\title{
Investigating the Effectiveness of Reactive Dispersants in Non-Aqueous Dispersion Polymerization
}

\author{
Weiwei Yang, Robin A. Hutchinson* \\ Department of Chemical Engineering, Dupuis Hall, Queen's University, Kingston, \\ Ontario K7L 3N6, Canada (robin.hutchinson@queensu.ca)
}

\begin{abstract}
Non-aqueous dispersion (NAD) radical polymerization is used to produce poly(acrylic) nanoparticles $(<200 \mathrm{~nm})$ at $60 \mathrm{wt} \%$ solids content by a starved-feed semibatch process, with steric stabilization provided by a low molecular weight vinyl-functionalized polymeric dispersant. The performance of two types of reactive dispersants are evaluated, a butyl methacrylate (BMA) based grafted dispersant with vinyl groups attached at random positions along the backbone, and a vinyl-terminated BMA macromer. The effectiveness of the dispersant, characterized by the amount of unreacted material remaining in solution and by NAD particle size, is dependent on the composition of the NAD being produced as well as the dispersant type and double bond content (macromer chain length). For an acrylate-rich recipe, the macromer dispersant is found to be incorporated more effectively than the randomly grafted dispersant, a result attributed to the uniform distribution of reactive double bonds across the entire dispersant molar mass distribution. The effectiveness of the macromer dispersant decreases when methacrylates are components of the NAD recipe.
\end{abstract}




\section{Introduction}

Non-aqueous dispersion (NAD) polymerization is a technique to produce nanometer-sized polymer particles at high solids content ( $\sim 60 \mathrm{wt} \%)$. These dispersions have been used since the 1960s as a component of solvent-borne automotive coatings formulations, added to prevent crack propagation and control the rheology upon application. ${ }^{[1]}$ Today NADs are also added to hybrid polymer coatings system in order to reduce solvent content while lowering energy consumption during the curing stage. The nanoparticles are of similar acrylic composition but of much higher molecular weight (MW) than the soluble polymer fraction of the formulation, and become uniformly crosslinked into the final topcoat or clear coat upon application without affecting optical clarity.

In dispersion polymerization, the system is initially homogenous but becomes heterogeneous as the reaction proceeds, since the continuous phase is a poor solvent for the polymer that is being produced. While there has been limited study of non-aqueous dispersions, much can be learned by examining the literature on particle formation in dispersion polymerizations conducted with common vinyl monomers like methyl methacrylate (MMA) or styrene (ST) in alcohol/water mixtures ${ }^{[2-4]}$ or supercritical carbon dioxide $\left(\mathrm{scCO}_{2}\right) \cdot{ }^{[5,6]}$ Fewer studies have been conducted in aliphatic hydrocarbon solvents such as hexane ${ }^{[7]}$ and dodecane, ${ }^{[8]}$ with shorter carbon chains (lower than butane) shown to be not effective. ${ }^{[9]}$ The solids content in these literature studies is normally at a significantly lower level $(<20 \mathrm{wt} \%)$ than that used in industrial NAD formulations (50-60 wt \%). Polar organic stabilizers with labile hydrogen atoms are often used in alcohol/water dispersions, such as poly(vinyl pyrrolidone), hydroxyl propyl cellulose, poly(acrylic acid), and poly(amic acid). ${ }^{[10-14]}$ These dispersants can become chemically attached to the polymer particles through $\mathrm{H}$-atom abstraction by growing oligomers to form in-situ grafts; a portion of the stabilizer may also be physically adsorbed onto the newly formed particles. ${ }^{[13]}$

Unlike the homopolymer dispersants in the polar solvent systems (alcohol/water), early studies with natural rubber or polybutadiene dispersants in non-aqeuous dispersions led to gel formation

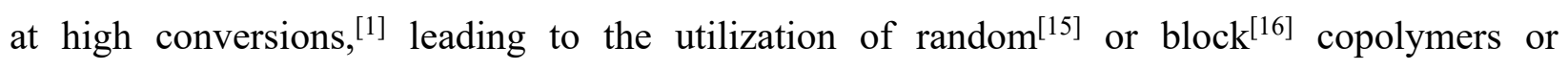
macromers $^{[8]}$ as steric stabilizers for non-polar NADs. A commercial macromer, methacryloxypropyl terminated poly(dimethylsiloxane) has been applied to make narrow distributed poly(methyl methacrylate) (pMMA) particles of $0.5-2.0$ microns in dodecane ${ }^{[8]}$ and in 
hexane; ${ }^{[7]}$ however, attachment of the dispersant to the particle surface was low, with only $10-20 \%$ of the dispersant added found attached to the particle surface despite the reactive end groups. ${ }^{[8]}$ Poly(12-hydroxystearic acid) (pHS) functionalized with reactive double bonds is also used as a reactive dispersant; ${ }^{[1,17]}$ however, as it is not completely soluble in non-polar solvents, ${ }^{[8]}$ the resultant polymer has also been reacted with MMA to form a comb (pHS-g-pMMA) copolymer in a multi-step synthesis process. ${ }^{[17],[18]}$ This dispersant was then used to produce $500-700 \mathrm{~nm}$ pMMA particles in dodecane with up to $50 \mathrm{wt} \%$ solids content; in this case, it is hypothesized that the dispersant physically, rather than covalently, attaches to the particle surface. ${ }^{[18]}$

Although the above studies are relevant, the particle sizes are generally larger than those required for coatings applications, with the objective of many of the studies to synthesize micron-sized particles with a very narrow size distribution for applications such as electrophoretic displays, ${ }^{[8]}$ support of biological materials ${ }^{[19]}$ and for chromatographic applications. ${ }^{[20]}$ Thus, the research has little concern for optimizing the usage of the dispersant in the system and for robustly producing dispersions with high polymer content and controlled viscosity, of paramount interest for automotive coating applications. In addition, the literature studies are conducted in batch, rather than the semibatch strategies practiced in industry for composition and temperature control.

In dispersion polymerization for coatings, ${ }^{[1]}$ a typical NAD formulation contains polymer content of 50-60 wt\%, with roughly a 1:2 ratio of stabilizer (dispersant) to core polymer, with particles typically less than $300 \mathrm{~nm}$. The soluble dispersant polymer, also an acrylic polymer but of lower polarity than the NAD core, is produced in a separate batch, sometimes involving multiple steps. Vinyl functionality is introduced to these low MW chains (weight-average molecular weight $\left(\mathrm{M}_{\mathrm{w}}\right)$ less than 10,000 Da) such that the molecules become covalently attached to the higher MW $\left(\mathrm{M}_{\mathrm{w}}\right.$ greater than 50,000 $\mathrm{Da}$, with crosslinking agents sometimes added) polar chains that precipitate out in the dispersion, thus preventing flocculation and controlling the average size of the polymer particles. It is possible that some of the dispersant also stabilizes by physical adsorption to the particle, and a significant portion of unreacted free dispersant remains in solution.

Despite these differences, there are important common features between academic and industrial dispersion systems. Particle nucleation, which occurs in the early stages of the reaction to control particle number and size, is not well understood. The polymeric dispersant is of critical importance, as it creates a reproducible polymerization system (unlike precipitation 
polymerization) by providing colloidal stability and helping to control average particle size and size distribution of the polymer produced. ${ }^{[1]}$ Solvent selection is also critical, with particle size and distribution greatly influenced by the composition. ${ }^{[21-23]}$

In this work, we systematically examine the semibatch non-aqueous dispersion polymerization of methyl methacrylate and methyl acrylate in heptane, using a functionalized low MW poly(butyl methacrylate) dispersant prepared in xylene. Using patent literature as a guide, ${ }^{[24]}$ this simplified recipe is used to produce low viscosity $60 \mathrm{wt} \%$ polymer dispersions with average particle size less than $200 \mathrm{~nm}$. Of particular interest is the role of the reactive dispersant, as we contrast the performance of material with randomly-distributed functionality to that of macromers with controlled functionality, as a means to improve the effective utilization of the dispersant.

\section{Experimental Section}

\section{Materials}

The monomers, n-butyl methacrylate (BMA, Sigma-Aldrich, 99\%), methacrylic acid (MAA, Sigma-Aldrich, 99\%), glycidyl methacrylate (GMA, Sigma-Aldrich, 97\%), methyl methacrylate (MMA, Sigma-Aldrich, 99\%) and methyl acrylate (MA, Sigma-Aldrich, 99\%), were used as received. The initiators, t-butyl peracetate (TBPA, Sigma-Aldrich, $50 \mathrm{wt} \%$ in mineral spirits) and 2, 2'-azobis-(2-methylbutyronitrile) (Vazo ${ }^{\circledR}$ 67, E. I. du Pont), were also used without any purification. The inhibitor catechol (Sigma-Aldrich, $\geq 99 \%$ ) and the amino catalyst, 2dimethylamino-2-methyl propanol (Sigma-Aldrich, $80 \mathrm{wt} \%$ in water), were utilized as received. Solvents used in the study, also without purification, were isopropanol (Sigma-Aldrich, 99.5\%), xylene (Sigma-Aldrich, $\geq 98.5 \%$ ), p-xylene (Sigma-Aldrich, $\geq 99 \%$ ), deuterochloroform ( $\mathrm{CDCl}_{3}$, Sigma-Aldrich, 99.8 atom\% D), heptane (Sigma-Aldrich, $\geq 99 \%$ ), and butyl acetate (SigmaAldrich, $\geq 99 \%$ ).

\section{Preparation of dispersant polymer}

All polymerizations were carried out in a $1 \mathrm{~L}$ semibatch reactor, which has been intensively used in our group. ${ }^{[25,26]}$ The recipe developed for this work is based upon patent literature, ${ }^{[24]}$ with a simplified copolymer formulation used (BMA based material with MAA added for functionality) rather than the 6 monomer system described in the patent. The operating procedure, however, was identical to the patent example, scaled to the $1 \mathrm{~L}$ reactor. $178.7 \mathrm{~g}$ xylene were added to the 
reactor and heated up to $138{ }^{\circ} \mathrm{C}$. A mixture containing of $409.7 \mathrm{~g} \mathrm{BMA}, 9.8 \mathrm{~g}$ MAA $(3.8 \mathrm{~mol} \%$ MAA with respect to total monomer), $22.5 \mathrm{~g}$ TBPA solution and $67.3 \mathrm{~g}$ xylene was pumped to the reactor at constant rate over $240 \mathrm{~min}$ and then held for another $30 \mathrm{~min}$; at this point, the conversion of the system is close to complete (>95\%). A mixture of $0.016 \mathrm{~g}$ catechol (to inhibit any further radical polymerization) in $0.18 \mathrm{~g}$ isopropanol was added, followed by $8.1 \mathrm{~g}$ GMA (a molar ratio of 0.5 relative to MAA) and $0.1 \mathrm{~g}$ 2-dimethylamino-2-methyl propanol (amine catalysis to promote reaction of the GMA epoxy group with the MAA carboxyl group). The system was held at reaction temperature for $120 \mathrm{~min}$ then cooled to room temperature. The final dispersant contained $\sim 60 \mathrm{wt} \%$ BMA-based polymer in xylene solvent. Three versions were produced, with the ratio of GMA:MAA set at $0,0.5$, and 1.0.

The BMA macromers, also 60-65 wt $\%$ in xylene, were kindly prepared and provided by Dr. Andrew P. Stamegna from Axalta Coating Systems.

\section{Non-aqueous dispersion polymerization}

MMA/MA copolymer and MA homopolymer systems were investigated as the core, with the conditions (temperature, addition rates, initiator amount, and ratio of dispersant to monomer) once again based upon patent literature. ${ }^{[24]}$ The reactor was charged with $128.2 \mathrm{~g}$ dispersant polymer solution and $130.1 \mathrm{~g}$ heptane, then heated to $92{ }^{\circ} \mathrm{C}$; heptane is a non-solvent for the more polar core polymer formed during the reaction, but is a good solvent for the BMA-based dispersant. A pre-mixed solution of $4.3 \mathrm{~g}$ butyl acetate and $0.32 \mathrm{~g}$ Vazo 67 was added, followed by addition over 210 min of a mixed solution consisting of $139.2 \mathrm{~g} \mathrm{MMA}, 65.8 \mathrm{~g} \mathrm{MA}, 48.7 \mathrm{~g}$ heptane, $64.4 \mathrm{~g}$ dispersant polymer solution and $2.5 \mathrm{~g}$ Vazo 67 . The dispersion was held at $92{ }^{\circ} \mathrm{C}$ for a further $45 \mathrm{~min}$, followed by addition of a solution of $0.82 \mathrm{~g}$ Vazo 67 and $9.8 \mathrm{~g}$ butyl acetate over $30 \mathrm{~min}$ and a further hold for $60 \mathrm{~min}$. The dispersion was cooled down to room temperature. Samples were taken at regular intervals during the reaction.

\section{Quantification of free dispersant}

The fraction of dispersant attached to the NAD nanoparticles was quantified by measuring the amount of the soluble polymer that remains in the continuous phase. $2 \mathrm{~g}$ of the NAD dispersion was diluted with $5 \mathrm{~mL}$ heptane. The mixture was centrifuged at $6000 \mathrm{rpm}$ for $10 \mathrm{~min}$ to separate the phases into a clear upper solution and a dense particle-rich layer. The clear liquid was collected, and the amount of polymer in solution was determined by gravimetry, leading to the 
estimate of winc., defined as the weight fraction of the incorporated dispersant; further details of this calculation are provided as Supporting Information. In addition, the soluble polymer recovered was characterized by proton nuclear magnetic resonance ( ${ }^{1} \mathrm{H}$ NMR) and size exclusive chromatography (SEC).

\section{Characterization}

${ }^{1} \mathrm{H}$ NMR was used to qualitatively verify the presence of reactive double bonds on the functionalized dispersant and quantitively measure the amount of the soluble polymer recovered from the NADs. Spectra were measured using a Bruker Acance-400 MHz spectrometer with automation on dried polymer dissolved in deuterated chloroform $\left(\mathrm{CDCl}_{3}\right)$.

Polymer MW averages and molar mass distributions (MMDs) were measured for all dispersants, for the soluble polymer recovered from the NADs, and for the complete NAD samples (mixed particle and continuous phases). The SEC analysis of lower MW samples (dispersants and soluble polymer) was done using a Waters 410 differential refractometer (DRI) operated at $35^{\circ} \mathrm{C}$, with THF as eluent at a flow rate of $0.3 \mathrm{~mL} \cdot \mathrm{min}^{-1}$. The DRI detector was calibrated by 10 narrow polystyrene (pST) standards with MW from $870 \mathrm{Da}$. to 355,000 Da. The MMDs for the NAD polymer (higher MW samples) were analyzed by SEC using a Viscotek 270 max separation module with triple detectors. THF was used an eluent at $1.0 \mathrm{~mL} \cdot \mathrm{min}^{-1}$ at $40{ }^{\circ} \mathrm{C}$. The reported results are based upon pST calibration, without further correction.

Particle average size and size distribution were measured on a Malvern Zeta-Sizer Nano ZS using dynamic light scattering (DLS) with an angle of $173{ }^{\circ}$ at $25^{\circ} \mathrm{C}$ in a quartz cuvette. Samples were diluted using a solvent mixture (50 $\mathrm{wt} \%$ heptane and $50 \mathrm{wt} \% \mathrm{p}$-xylene), with the refractive index (1.418) and viscosity $(0.562 \mathrm{cP})$ of the binary solvent mixture calculated via the LorentzLorenz formula ${ }^{[27]}$ and the Grunberg and Nissan equation, ${ }^{[28]}$ respectively. The refractive index of polymer dispersion is 1.59 , as provided by Axalta Coating Systems. Viscosities were measured at room temperature using a Brookfield Viscometer with a UL adapter at a rotation rate of 0.5 to $1.0 \mathrm{rpm}$. 


\section{Results and Discussion}

\section{NAD synthesis with grafted dispersant}

Controlling the number of functional vinyl groups per dispersant polymer chain is important to have a soluble and effective stabilizer ${ }^{[1]}$ Conventional "grafted" dispersants are made by starved-feed free radical polymerization to produce copolymers with carboxyl groups, which then are functionalized through an amine-catalyzed reaction with the epoxy group of glycidyl methacrylate (GMA) to produce a functional unsaturated dispersant, as shown in Figure $1 a^{[24,29]}$ As summarized in Table 1, the dispersants synthesized in this study all have the same base composition ( $\mathrm{M}_{\mathrm{n}}$ of $\sim 4500 \mathrm{Da}$ for an average chain length of 30 monomer units, with $4 \mathrm{~mol} \%$ MAA for an average of 1.0 MAA units per chain), but have been reacted with differing levels of GMA to vary the level of functionalization of the chain. Assuming quantitative reaction, the average number of double bonds per chain for Dispersant I, Dispersant II and Dispersant II is 0, 0.5 and 1.0, respectively. As described in the Supplemental Information section, the presence of the double bond in the dispersant after the esterification is confirmed by ${ }^{1} \mathrm{H}$ NMR, as has been observed in literature. ${ }^{[30]}$ With half of the carboxyl groups functionalized, Dispersant II is similar to the examples found in the patent literature; ${ }^{[24]}$ Dispersant I (non-functionalized) and Dispersant III (fully-functionalized) were synthesized to further investigate the role of the functional groups during NAD synthesis. 
(a)

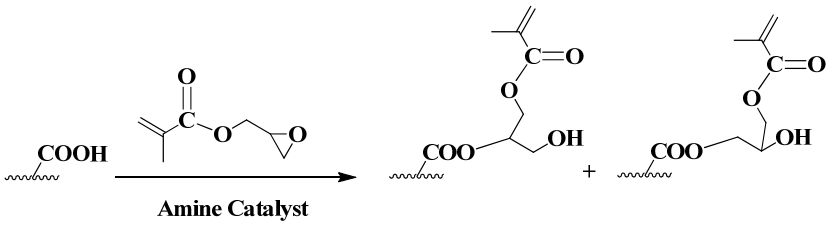

(b)

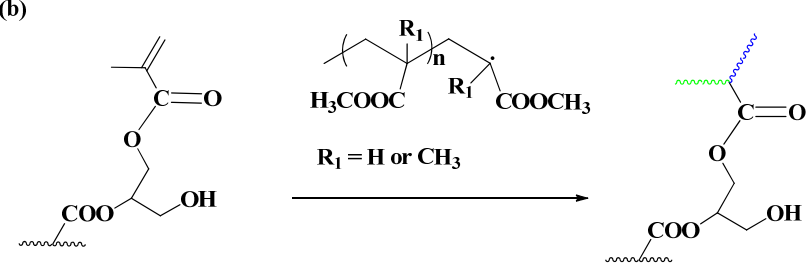

(c)

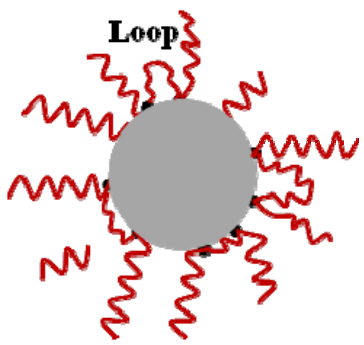

Figure 1. Schematic of reactions involved in the synthesis of NAD prepared with grafted dispersant: (a) esterification between GMA and MAA units to introduce reactive functionality to the polymer dispersant; (b) addition of the vinyl-functionalized dispersant to MMA or MA radicals present during NAD synthesis; (c) proposed attachment of dispersant to the particle surface, with multiple functionalities per chain leading to the potential of multiple surface attachments, loop formations and crosslinks.

Table 1. pBMA-based grafted dispersants produced with varying GMA to MAA molar ratio.

\begin{tabular}{llll}
\hline Sample & GMA/MAA molar ratio & $\mathrm{M}_{\mathrm{n}}(\mathrm{Da})$ & $\mathrm{M}_{\mathrm{w}}(\mathrm{Da})$ \\
\hline Dispersant I & 0 & 4345 & 7534 \\
Dispersant II & 0.5 & 4812 & 9571 \\
Dispersant III & 1.0 & 4776 & 9261 \\
\hline
\end{tabular}

The dispersant solutions, consisting of $60 \mathrm{wt} \%$ polymer in xylene solution, are stored and used as a reactive component to produce polymeric nanoparticles in non-aqueous dispersion (NAD), also by a starved-feed semibatch process. Figure $1 \mathrm{~b}$ is a schematic of the reaction of the grafted dispersant with the radicals (MMA or MA) in the system during NAD copolymerization. As 
described in the experimental section, two thirds of the dispersant is pre-charged to the reactor (along with solvent), and the remaining amount fed with monomer, initiator and additional solvent over a $3.5 \mathrm{~h}$ period. The final NAD low viscosity mixture (easily pourable) contains 60 $\mathrm{wt} \%$ polymer in xylene/heptane solvent, of which $20 \mathrm{wt} \%$ of the polymer is the original dispersant, and the remaining $40 \mathrm{wt} \%$ is polymer formed by complete conversion of the MA/MMA monomer into polymer particles. The more polar MA/MMA copolymer precipitates out of the non-polar heptane solvent as it is formed, with the nanoparticles stabilized by the reactive dispersant. Images of NAD samples taken in the polymerization period are shown in Figure 2. The solution is still clear at $20 \mathrm{~min}$, then turns translucent ( $40 \mathrm{~min}$ ) to milky and opaque after $60 \mathrm{~min}$. Nucleation of polymeric particles formed by dispersion polymerization in polar media occurs at the early stages of polymerization, ${ }^{[31]}$ and in batch systems the number of particles remains constant after nucleation, with newly generated small particles (or polymer chains) swept up by existing particles. ${ }^{[23]}$ Figure 3 demonstrates that particle size remained relatively constant in the semibatch system after $120 \mathrm{~min}$; unfortunately, it was difficult to obtain reliable data during the earlier nucleation stage of the reaction. Although the particle size and concentration remains constant, the total number of particles increases over the feeding period, likely due to the concurrent addition of dispersant solution with monomer (see Supporting Information).

Varying the level of functionalization on the grafted dispersant helps to provide a clearer picture of its role during particle formation. As seen in Figure 2, the formation of particles in NAD I is delayed, as there are no double bonds by which Dispersant I can become covalently incorporated to the MA/MMA copolymer particles. The influence of the level of functionalization on the properties of the final NAD product is summarized in Table 2. The amount of Dispersant I attached (or physically adsorbed) to the particles is only $13 \%$, as quantified by performing gravimetry on the continuous phase after destabilizing the dispersion to separate out the particles. Introduction of the reactive double bonds to the polymer increases the fraction incorporated to $32 \%$ for NAD II (made with Dispersant II) and 44\% for NAD III (made with Dispersant III). The effect on dispersant incorporation on average particle size is clear, as seen by the corresponding decrease from 237 (NAD I) to 162 (NAD II) to $147 \mathrm{~nm}$ (NAD III). 

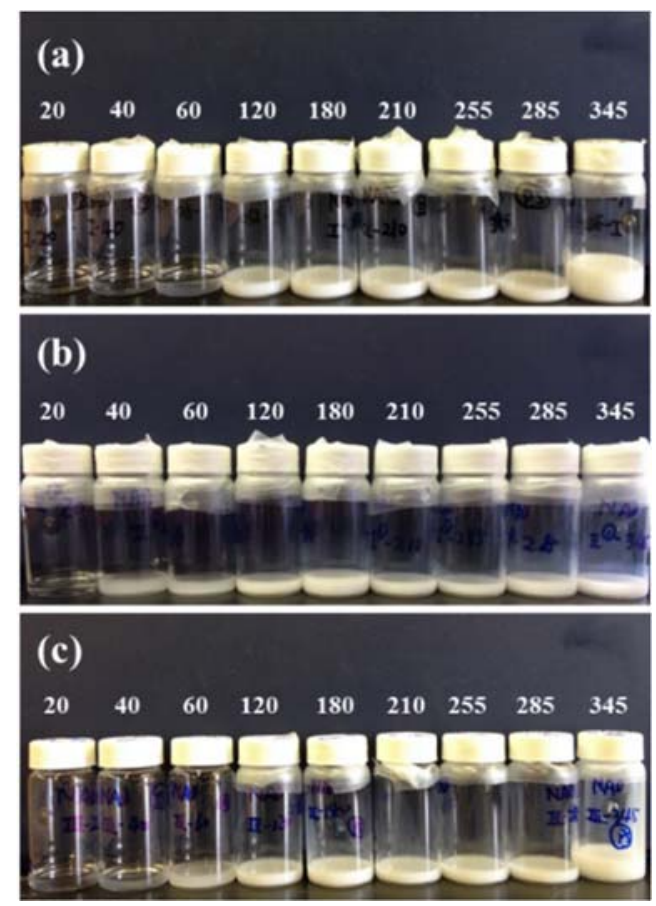

Figure 2. Photographs of non-aqueous dispersion samples taken during the reaction period, with sample time (min) indicated above the individual vials: (a) NAD I; (b) NAD II; (c) NAD III.

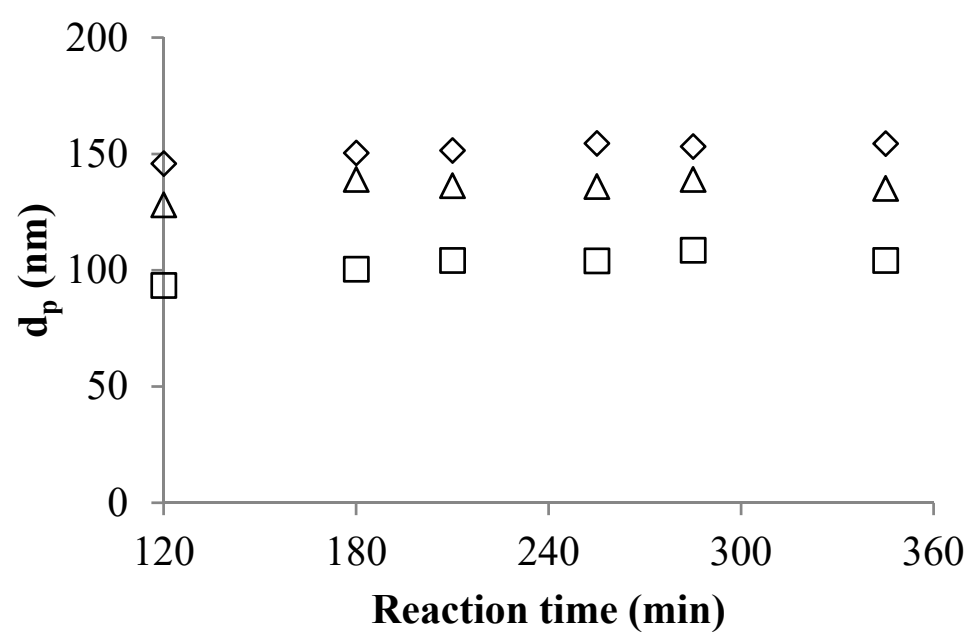

Figure 3. The evolution of particle size during the semibatch production of NAD II (diamond), NAD B-M2 (square), and NAD B-M5 (triangle).

These results suggest that increased functionalization is a viable means to decrease particle size while also increasing the utilization of the dispersant. (Other approaches, such as recipe modifications are being studied, but will not be discussed in this publication.) However, there is 
a practical limitation to this approach. While starved-feed reactor operation provides excellent control of the average number of MAA units incorporated per dispersant chain (and thus double bonds per chain via epoxidization), the stochastic nature of radical polymerization means that even under perfect control of overall copolymer composition, a significant fraction of the polymer chains produced contains no functionality. A recent simulation study ${ }^{[32]}$ demonstrates that most of the chains without functionality are, not surprisingly, at the low MW side of the distribution, and that many of the chains on the high MW side of the distribution contain multiple functional groups per chain. This stochastic variability is quite important when producing low MW functional polymers: the simulation study indicated that for a numberaverage chain length of 20 and a single functional group per chain (on average), only a quarter of the chains produced have the desired functionality of one unit per polymer molecule, with close to half of the chains produced containing no functional groups. ${ }^{[32]}$

Our experimental findings illustrate the importance of functional group distribution in the dispersants. Figure 4 compares the molar mass distributions (MMDs) of the NAD product with those of the dispersants used in the reaction. The MMDs are bimodal, with the low-MW mode overlapping the MMDs of the original dispersant, indicating that a high fraction of the dispersant has not reacted. It can also be seen that the NAD II and NAD III polymers have an observable increase of material in the high MW tail, as also reflected in the weight-average $\left(M_{\mathrm{w}}\right)$ values summarized in Table 2. Note that it was not possible to measure the MMD of the final NAD III sample, as the polymer did not fully dissolve in THF; thus the MMD shown (and $M_{\mathrm{w}}$ reported) is from a sample collected earlier in the reaction. We hypothesize that NAD III is partially crosslinked, due to the reaction of multiple double bonds located on the higher MW Dispersant III chains, as illustrated schematically in Figure 1c. The chemical attachment of dispersant to the particle surface is dependent on the number and location of the functional groups, with more than one attachment likely formed for the higher MW dispersant. Although the formation of crosslinking may not affect the product application (some core systems are crosslinked by design ${ }^{[24]}$ ), the efficacy of the dispersant may be lessened due to the loops formed with multiple attachments of the chain to the particle surface. ${ }^{[33]}$ 
Table 2. Properties of NAD polymer synthesized using grafted dispersants with varying double bond content (GMA/MAA molar ratios, see Table 1).

\begin{tabular}{lllcll}
\hline Sample & $\begin{array}{c}\text { GMA/MAA } \\
\text { molar ratio }\end{array}$ & $\mathrm{M}_{\mathrm{n}}(\mathrm{Da})$ & $\mathrm{M}_{\mathrm{w}}(\mathrm{Da})$ & $w_{\text {inc }}$ & \\
\hline NAD I & 0.0 & 12055 & 60347 & 0.131 & $\mathrm{~d}_{\mathrm{p}}(\mathrm{nm})^{\mathrm{b}}$ \\
NAD II & 0.5 & 12254 & 92705 & 0.320 & 162 \\
NAD III & 1.0 & $10479^{\mathrm{a})}$ & $110268^{\mathrm{a}}$ & 0.440 & 147
\end{tabular}

a) Sample collected at $285 \mathrm{~min}$; gel formed for sample at $390 \mathrm{~min}$ (final product).

b) $w_{\text {inc }}$ is the weight fraction of dispersant that is attached to the particle, as determined from analysis of the amount of polymer remaining in solution; $d_{\mathrm{p}}$ is the average particle size, as measured by DLS

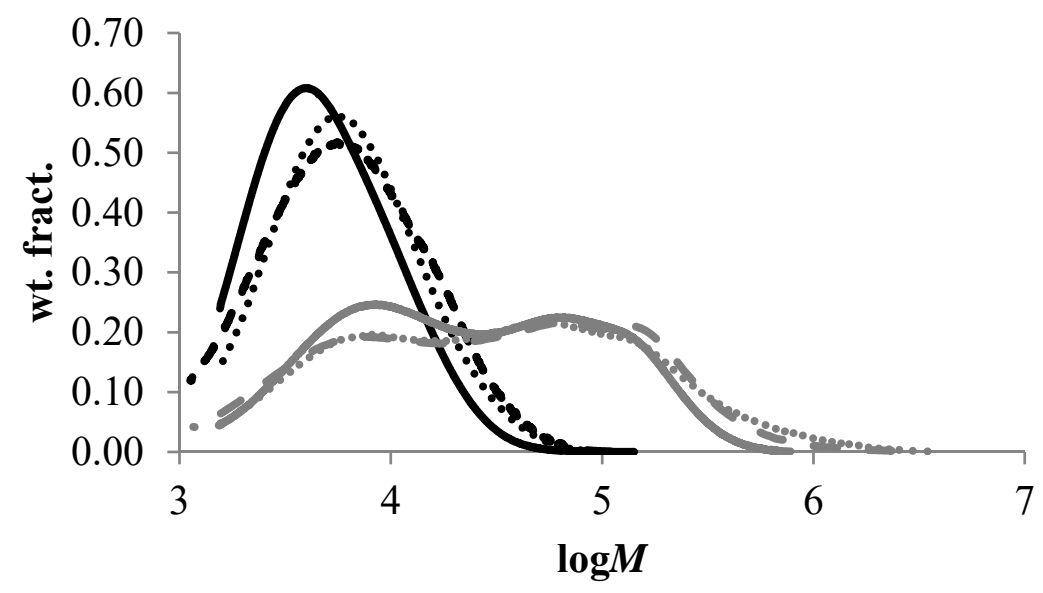

Figure 4. Polymer MMDs of dispersants (black lines) and the corresponding product produced by non-aqueous dispersion (NAD) polymerization (grey lines): Dispersant I/NAD I (solid lines), Dispersant II/NAD II (dashed lines) and Dispersant III/NAD III (dotted lines).

The soluble polymer that remains in the continuous phase has been separated out from the NAD product for further analysis. NMR analysis (see Supporting Information), shows that this material is predominantly $(>95 \mathrm{wt} \%)$ the original BMA-based dispersant, although some MMA/MA soluble copolymer is also found. The proton signal from the dispersant double bonds is no longer observable by NMR, suggesting that the chains that remain in solution are largely 
unreactive. This finding can also be inferred by comparing the MMDs of the soluble polymer to that of the original dispersant. The distributions from NAD II and NAD III soluble fractions are shifted to lower MW (loss of higher MW tail) compared to Dispersant II and Dispersant III, respectively, as observed in Figure 5b-c. In contrast, the MMD of the soluble polymer from NAD I is exactly the same as the non-functionalized Dispersant I (Figure 5a).
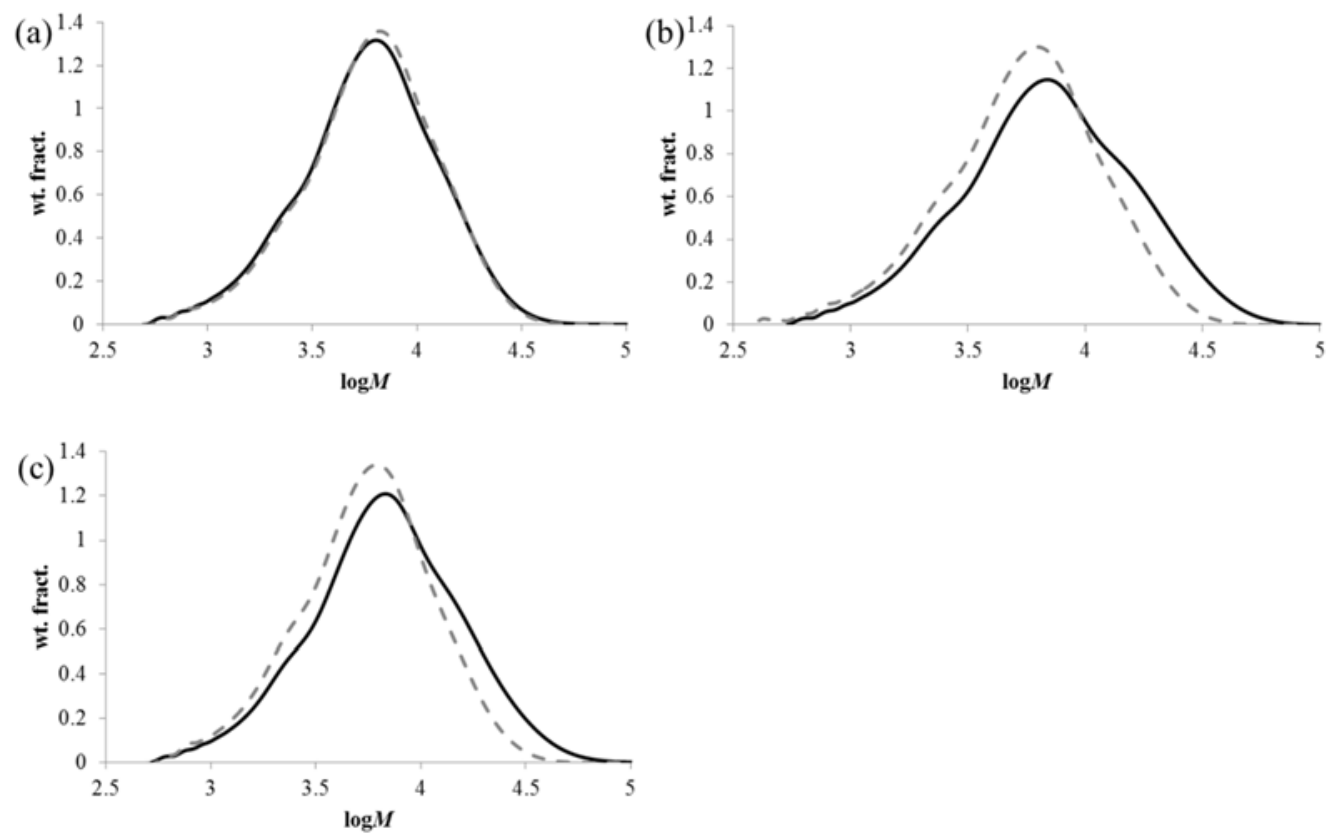

Figure 5. MMDs of polymer dispersants (solid lines) and soluble polymer fraction isolated from NADs (dashed line): (a) Dispersant I/NAD I; (b) Dispersant II/NAD II; (c) Dispersant III/NAD III.

These results provide a strong indication of the uneven distribution of vinyl groups in the grafted dispersant produced by free radical polymerization. The higher MW chains contain a greater number of the reactive double bonds, and thus are preferentially removed from solution by reaction with the NAD core polymer. Thus, while increasing the double-bond content of the dispersant leads to smaller particles and an increased utilization of the dispersant, it can also lead to the formation of crosslinked polymer due to the higher probability of having multiple functionalities per chain. The lower MW chains, more likely to contain no functionality, are not available for reaction and thus are less effective at stabilizing the particles. While the dispersant system can form stable NAD products, there is opportunity for improvement. 


\section{NAD Synthesis with Macromer Dispersant}

We hypothesized that the efficacy of the dispersant can be increased if it is possible to ensure that every chain contains exactly one reactive double bond, and if that bond is located at the end of the chain such that the stabilizer molecule extends out from the particle surface after being incorporated (Figure 6c). Thus a series of butyl methacrylate macromers have been synthesized by cobalt chain transfer (CCT) polymerization (Figure 6a) and tested as potential dispersants for NAD polymerization. CCT is a well-understood technique to produce low molecular weight (MW) polymer chains at relatively low temperatures using ppm amounts of catalytic chain transfer agent without incorporation of the transfer agent into the macromolecules formed. Under well-chosen conditions, nearly all of the polymer chains contain an unsaturated bond at the chain end, such that these polymers can be used as macromers in subsequent polymerization. ${ }^{[34,35]}$ The macromers used in this work were prepared as 60-65 $\mathrm{wt} \%$ pBMA solution in xylene, so as to provide a direct comparison to the grafted dispersant. As summarized in Table 3, a range of chain lengths were studied, with the SEC-measured number-average MW value confirmed by NMR analysis (see Supporting Information).

A series of NAD polymerizations were conducted using these macromer dispersants in place of the grafted dispersants for the same MMA/MA NAD core recipe, with results summarized in Table 3. Compared to the NAD particles produced with grafted dispersant (150-160 nm), the NAD particles produced with the macromers are smaller, with an average $d_{p}$ as low as $100 \mathrm{~nm}$ (see Figure 3). As shown in Figure 7a-b, the particles are not evident until the 60 min sample is collected, in contrast to the situation with grafted dispersant (Figure 2). The samples at this point still have a bluish tinge, indicative of the smaller particles in the system. 
(a)
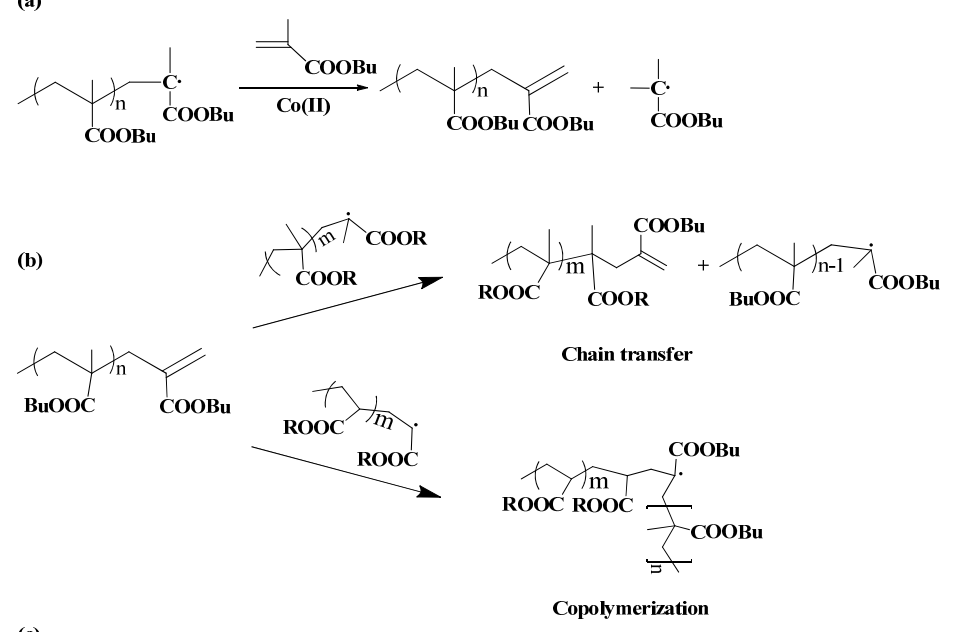

(c)

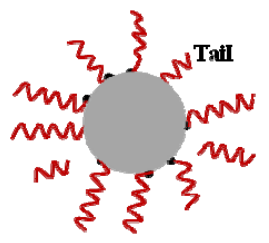

Figure 6. Schematic of reactions involved in the synthesis of NAD prepared with macromer dispersant: (a) Macromer synthesis by cobalt chain transfer polymerization; (b) macromer reaction with methyl methacrylate radical (chain transfer) and methyl acrylate radical (copolymerization); (c) proposed attachment of macromer to the particle surface, with a single attachment formed at the chain end.

Table 3. Properties of butyl methacrylate macromers (M1 through M6) and corresponding NAD copolymer (NAD B-M1 through NAD B-M6) synthesized with macromer dispersants

\begin{tabular}{c|lc|lllc}
\hline \multirow{2}{*}{ Sample } & \multicolumn{3}{|c|}{ Macromer MWs } & \multicolumn{4}{c}{ NAD Properties } \\
& $\mathrm{M}_{\mathrm{n}}(\mathrm{Da})$ & $\mathrm{M}_{\mathrm{w}}(\mathrm{Da})$ & $\mathrm{M}_{\mathrm{n}}(\mathrm{Da})$ & $\mathrm{M}_{\mathrm{w}}(\mathrm{Da})$ & $w_{\text {inc }}$ & $\mathrm{d}_{\mathrm{p}}(\mathrm{nm})$ \\
\hline NAD B-M1 & 5259 & 9056 & 12521 & 38338 & 0.400 & 99.7 \\
NAD B-M2 & 5560 & 9249 & 12214 & 42915 & 0.414 & 104.2 \\
NAD B-M3 & 5684 & 9625 & 12027 & 43896 & 0.405 & 118.2 \\
NAD B-M4 & 6252 & 10953 & 12821 & 44147 & 0.399 & 126.8 \\
NAD B-M5 & 7059 & 11982 & 14063 & 52704 & 0.357 & 135.2 \\
NAD B-M6 & 8315 & 15006 & 14553 & 61302 & 0.344 & 168.9 \\
\hline
\end{tabular}

$W_{\text {inc }}$ is the weight fraction of dispersant that is attached to the particle, as determined from analysis of the amount of polymer remaining in solution; $d_{\mathrm{p}}$ is the average particle size, as measured by DLS. 


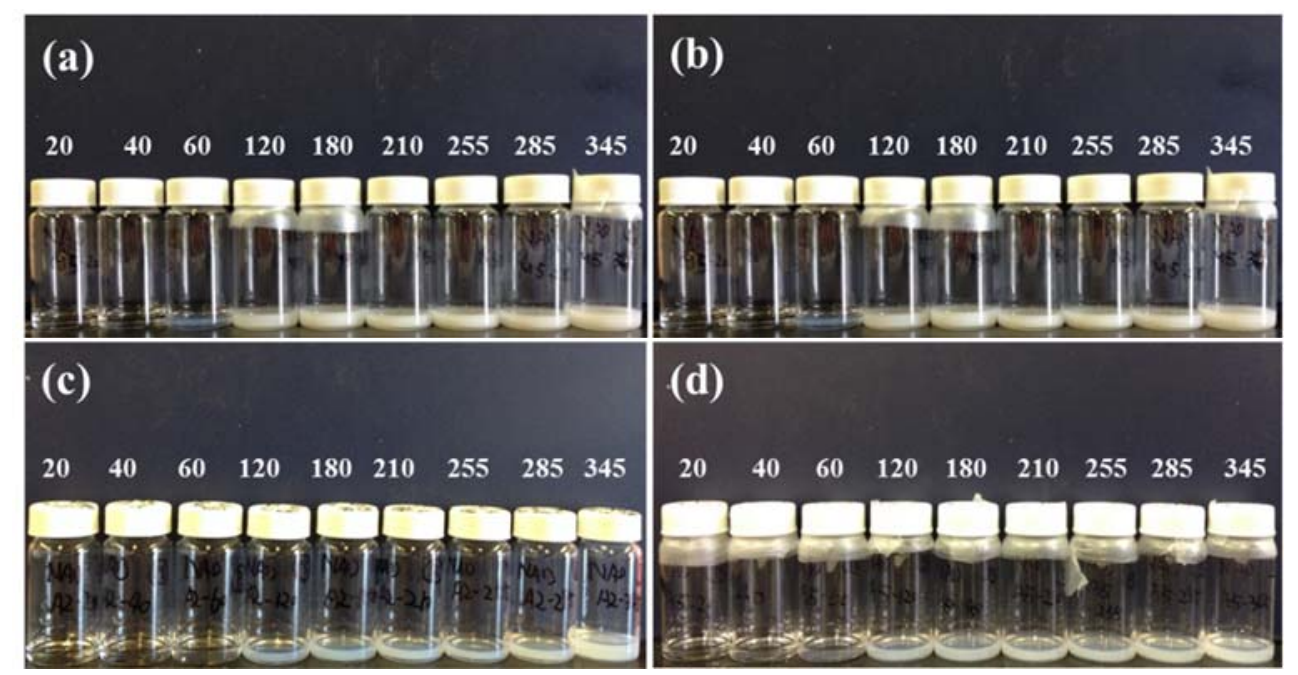

Figure 7. Photographs of NAD samples taken during the reaction period, with sample time (min) indicated above the individual vials: (a) NAD copolymer synthesized with M2; (b) NAD copolymer synthesized with M5; (c) NAD acrylate homopolymer synthesized with M2; (d). NAD acrylate homopolymer synthesized with M5.

As shown in Figure 8, there is a direct correlation between particle size and macromer $\mathrm{M}_{\mathrm{n}}$; the lower-MW M1-M3 dispersants have a higher double bond content per unit mass, and thus are incorporated more readily into the growing MMA/MA copolymer chains. As the MW values of the grafted and macromer dispersants are similar, it can be concluded that the improved stabilization is due to the even distribution of the double bonds (one per chain) among the macromer molecules compared to the grafted dispersant. Moreover, as the double bond is located at the end of the chain, we hypothesize that the stabilizer chains attached to the NAD particles extend out in solution (Figure 6c), in contrast to the attachment of the grafted dispersant (with randomly distributed double bonds) attached to the NAD particles (Figure 1c).

Despite the decrease in particle size, the fraction of the macromer dispersant that is incorporated (or adsorbed) onto the NAD particles is $30-42 \mathrm{wt} \%$, with the majority of the dispersant remaining in the continuous phase. This result was surprising, as it was expected that the even distribution of double bonds in the macromer would lead to improved dispersant incorporation compared to the grafted dispersant. In addition, as summarized in Table 3, the average MWs values of the NAD polymer produced are significantly lower $\left(\mathrm{M}_{\mathrm{w}}\right.$ values of $\left.4-6 \times 10^{4} \mathrm{Da}\right)$ than those of the polymer produced with the grafted dispersants (Table 2); as shown in Figure 9, it was found that the average MW of the polymer produced decreased as the MW of the macromer dispersant is decreased. 


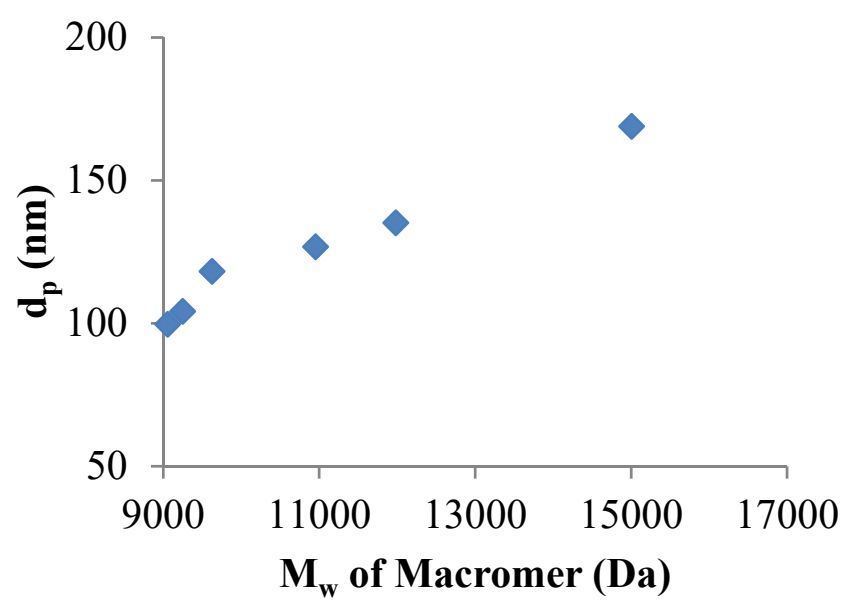

Figure 8. Average particle size $(\mathrm{nm})$ produced by NAD MMA/MA copolymerization as a function of the weight-average MW of the macromer used as dispersant.

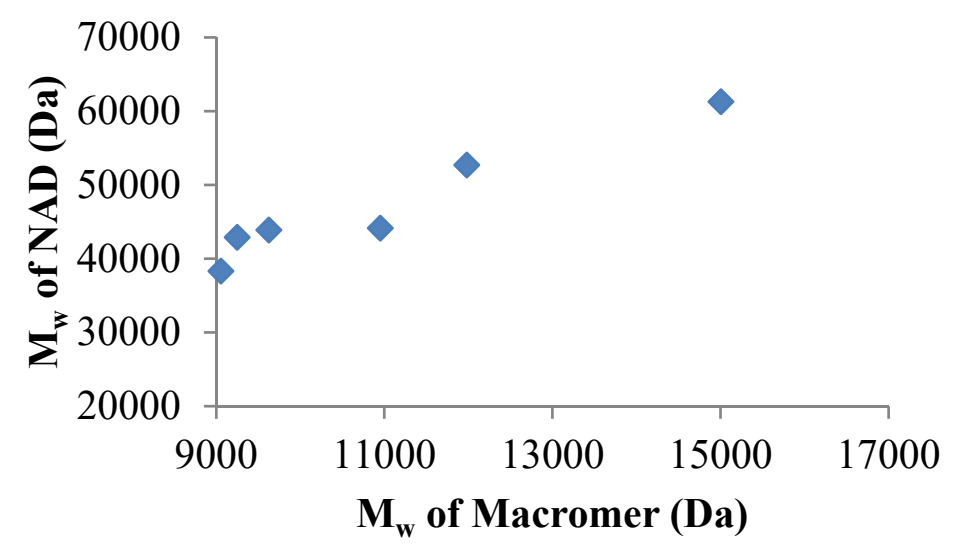

Figure 9. Weight-average MW of polymer produced by NAD MMA/MA copolymerization as a function of the weight-average MW of the macromer used as dispersant.

As shown in Figure 10, the MMDs of the NAD polymer remain bimodal, with the lower MW peak corresponding to the MMD of the macromers used as dispersants. The soluble polymer recovered from the reactions was confirmed to be predominantly ( $>95 \mathrm{wt} \%$ ) unreacted dispersant that, according to NMR analysis, contained reactive double bonds (see supplemental information). In addition, the MMD of the soluble polymer fraction was found to overlay the distribution of the original macromer within experimental error, as shown for M2 and M5 in Figure 11. (MMD plots for the complete set of macromer, NAD, and soluble polymers samples followed the same trends as those shown in Figures 10 and 11.) 


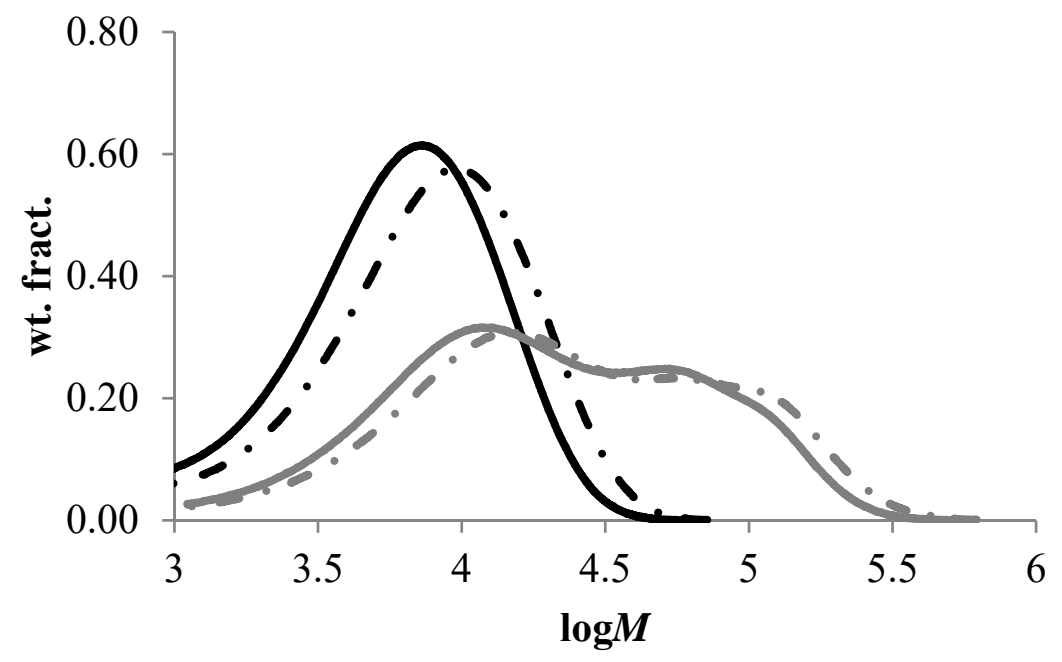

Figure 10. Polymer MMDs of macromer dispersants (black lines) and the corresponding product produced by non-aqueous MMA/MA dispersion (NAD) copolymerization (grey lines) for M2/NAD B-M2 (solid lines) and M5/NAD B-M5 (dot-dash lines).

All of these results indicate that, although complete conversion of the MMA and MA monomers are achieved, the "conversion" (incorporation) of the macromer during NAD formation is significantly lower. This result can be attributed to the lower reactivity of macromers: while the double bond of the grafted dispersant is part of an ester moiety along the chain (Figure 1a), the double bond of the macromer is at the terminus, with the polymer chain replacing the $\mathrm{CH}_{3}$ group of a methacrylate (Figure 6a). It has been documented that this type of macromer does not homopolymerize or copolymerize with methacrylates because of the competing $\beta$-scission (chain-transfer) reaction. ${ }^{[36]}$ However, copolymerization of macromer with acrylates readily occurs, such that the macromer is incorporated into the chain as a graft. ${ }^{[36]}$ Both reactions must occur for the methacrylate/acrylate recipe used in this work, as shown in Figure $6 \mathrm{~b}$ : incorporation as a stabilizing graft when reacting with an MA radical, and acting as a chain transfer agent (hence reducing polymer MW) when interacting with an MMA radical. 

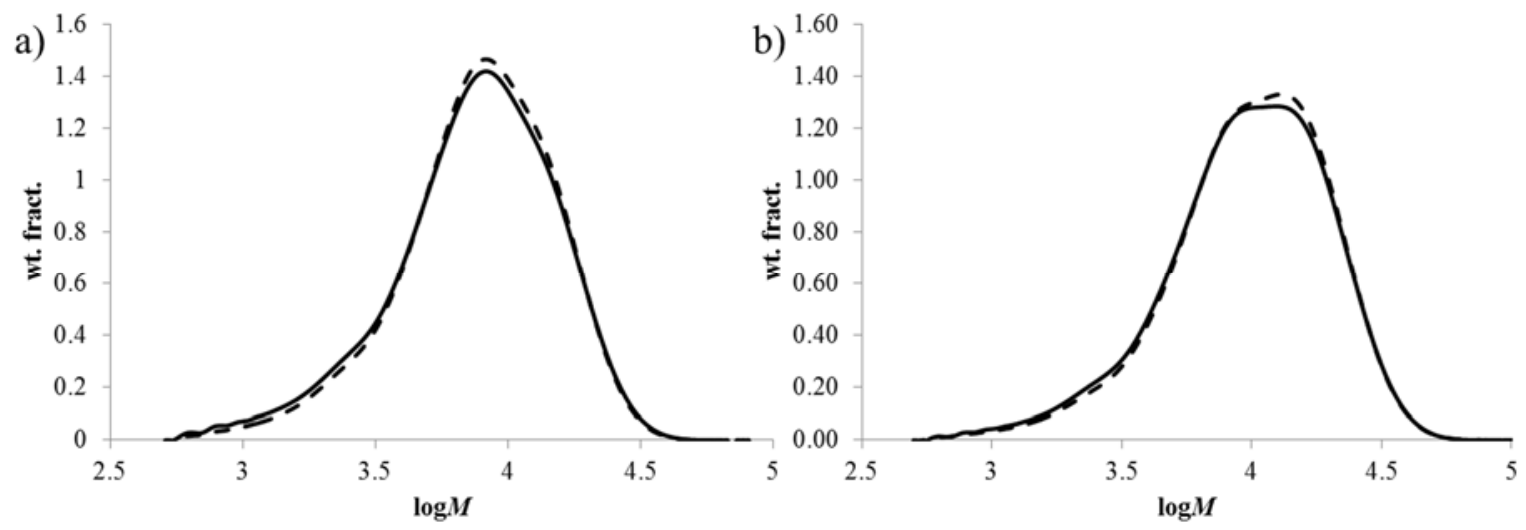

Figure 11. MMDs of polymer dispersants (solid lines) and soluble polymer fraction isolated from NADs (dashed line): a) M2 and NAD B-M2; b) M5 and NAD B-M5.

\section{Grafted vs. Macromer Dispersant (Acrylate Homopolymer Core)}

To further explore the replacement of grafted dispersant with macromer, the performance of Dispersant II was compared to two of the macromers (M2 and M5) for NAD produced using only methyl acrylate as the core. According to the mechanisms discussed above, this should eliminate the chain-transfer reaction that occurs between macromer and methacrylate radicals (no longer present in the system), and thus increase dispersant efficiency.

Table 4. Comparison of the properties of NADs with MMA/MA copolymer core and MA only core, synthesized using both grafted dispersant and macromer

\begin{tabular}{llllllll}
\hline Dispersant & Core & Sample & $\mathrm{M}_{\mathrm{n}}(\mathrm{Da})$ & $\mathrm{M}_{\mathrm{w}}(\mathrm{Da})$ & Winc & $\mathrm{d}_{\mathrm{p}}(\mathrm{nm})$ & Viscosity $(\mathrm{cP})$ \\
\hline Grafted & MMA/MA & NAD II & 12254 & 92705 & 0.32 & 162 & 85 \\
(Disp II) & MA only & NAD A-II & 11159 & 86769 & 0.46 & 109 & 236 \\
\hline M2 & MMA/MA & NAD B-M2 & 12214 & 42915 & 0.41 & 104 & 154 \\
& MA only & NAD A-M2 & 16037 & 81541 & 0.61 & 70 & 199 \\
\hline M5 & MMA/MA & NAD B-M5 & 14063 & 52704 & 0.36 & 135 & 117 \\
& MA only & NAD A-M5 & 16227 & 71054 & 0.59 & 83 & 874 \\
\hline
\end{tabular}

$W_{\text {inc }}$ is the weight fraction of dispersant that is attached to the particle, as determined from analysis of the amount of polymer remaining in solution; $d_{\mathrm{p}}$ is the average particle size, as measured by DLS. 
Pictures taken for NAD A-M2 and NAD A-M5 are shown in Figure 7c and 7d, with the bluish tingle still seen at 120 min indicating a formation of even smaller particles. The results of these experiments are summarized in Table 4, which also includes a summary of MMA/MA NAD produced with the same three dispersants. There are several key points demonstrated by these results. First of all, while the MW averages are the same for the MA homopolymer and the MMA/MA NAD produced with the grafted dispersant, there is a significant difference for the reactions run with macromer as dispersant. This result is consistent with the hypothesis that the macromer acts as a chain transfer agent rather than as a comonomer with methacrylate radicals and copolymerizes only with acrylate. ${ }^{[36]}$ Thus, the incorporation of the macromer to the acrylate homopolymer core is higher, up to $60 \%$, compared to the $30-42 \%$ incorporation when MMA is a component in the recipe. With the increased effectiveness of the dispersant, the average particle size decreases to $70 \mathrm{~nm}$ for M2 and $83 \mathrm{~nm}$ for M5; the smaller particle size produced with M2 is due to the higher concentration of double bonds (lower $\mathrm{M}_{\mathrm{n}}$ ) compared to M5 for the same mass of dispersant added. It is interesting to note that a higher portion of the grafted dispersant is also incorporated with the core for the acrylate homopolymer (with smaller particle size) compared to the copolymer system. This result is not fully understood, but may be related to the higher polarity of MA compared to MMA. There is also a small increase in the viscosity of NADs prepared with the MA only core compared to the copolymerization. While undoubtedly related to particle size, the increase in viscosity is larger with M5 compared to the other dispersants. However, in all cases the NAD produced is stable and pourable. What is clear is that the uneven distributed vinyl group among Dispersant II makes it less efficient than M2, even for the base case methacrylate/acrylate recipe. Although the two polymers have similar MWs (see Table 1 and Table 3), M2 has one double bond per chain, while Dispersant II has an average of 0.5 double bonds per chain, with a broad distribution of these double bonds across the MMD.

\section{Conclusions}

NAD products with high solids content $(\sim 60 \mathrm{wt} \%)$ and small particle size $(<200 \mathrm{~nm})$ were produced via starved-feed semibatch polymerization employing two types of reactive polymeric low-MW pBMA-based dispersants. The grafted dispersant was synthesized with the vinyl groups randomly distributed along the backbone, with many low MW chains unfunctionalized. Thus, the effectiveness of the dispersant, as characterized by amount incorporated to the particle and average particle size, is lower than the (vinyl-terminated) macromers synthesized by BMA 
polymerization in the presence of a cobalt chain transfer agent. Although the efficacy of the grafted dispersant could be improved by increasing the average double-bond content per chain, the presence of multiple functional groups per chain led to crosslinking in the NAD system. Thus, it can be concluded that the macromers provide more efficient stabilization than the grafted dispersant, with the lowest MW macromers $\left(\mathrm{M}_{n}\right.$ of 5000-6000 Da) most effective due to the higher double-bond content per unit mass.

In the presence of MMA (MMA/MA copolymer core), lower efficacy was found for both types of dispersants compared to NAD produced with an MA homopolymer core. With macromer dispersant, the standard recipe produced particles of $<100 \mathrm{~nm}$ in size, with up to $60 \mathrm{wt} \%$ of the dispersant incorporated into the particle phase. The improved effectiveness is hypothesized to be partly due to the increased polarity of MA. However, the chain-transfer reaction that occurs with methacrylate radicals is eliminated in the acrylate homopolymerization system, thus increasing the effectiveness of macromer as a dispersant and increasing the MW of the NAD polymer. Future work will focus on evaluating the effectiveness of macromer as dispersant for more complex multi-monomer recipes, as well as examining the influence of dispersant feeding profile on its performance.

Acknowledgement. The authors acknowledge the valuable input from Drs. Robert J. Barsotti, Andrew P. Stamegna and Jeffery W. Johnson of Axalta Coating Systems. This work was financially supported by Axalta Coating Systems (Wilmington, U.S.A) and the Natural Sciences and Engineering Research Council of Canada.

\section{References}

1. R. Dowbenko, D. P. Hart, Ind. Eng. Chem. Prod. Res. Develop. 1973, 12, 14.

2. C. M. Tseng, Y. Y. Lu, M. S. El-Aasser, J. W. Vanderhoff, J. Polym. Sci. Part A: Polym. Chem. 1986, 24, 2995.

3. A. J. Paine, W. Luymes, J. McNulty, Macromolecules 1990, 23, 3104.

4. M. S. Kim, G. H. Lee, J. Hong, H. Lee, Mat. Sci. Eng. C 2007, 27, 1247.

5. Q. Dong, Y. Liu, J. Appl. Polym. Sci. 2004, 92, 2203. 
6. H. Yuvaraj, H. S. Hwanga, Y. S. Junga, J.-H Kima, S.-S Hongb, K. T. Lima, J. Supercrit. Fluid. 2007, 42, 351.

7. S. M. Klein, V. N. Manoharan, D. J. Pine, F. F. Lange, Colloid. Polym. Sci. 2003, 282, 7.

8. A. P. Richez, L. Farrand, M. Goulding, J. H. Wilson, S. Lawson, S. Biggs, O. J. Cayre, Langmuir 2014, 30, 1220.

9. I. Tausendfreund, F. Bandermann, H. Siesler, M. Kleimann, Polymer 2002, 43, 7085.

10. A. M. I. Ali, P. Pareek, L. Sewell, A. Schmid, S. Fujii, S. P. Armes, I. M. Shirley, Soft Matter 2007, 3, 1003.

11. W. Yang, D. Yang, J. Hu, C. Wang, S. Fu, J. Polym. Sci. Part A: Polym. Chem. 2001, 39, 555 .

12. V. L. Covolan, P. L. O. Volpe, S. G. C. Castro, R. Landers, E. G. Fernandes, G. Ruggeri, Macromol. Chem. Phys. 2002, 203, 1454.

13. S. Shen, E. D. Sudol, M. S. El-Aasser, J. Polym. Sci. Part A: Polym. Chem. 1994, 32, 1087.

14. S. Watanabe, K. Ueno, M. Murata, Y. Masuda, Polym. J. 2006, 38, 471.

15. K. E. J. Barrett, Brit. Polym. J. 1973, 5, 259.

16. J. V. Dawkins, G. G. Maghami, S. A. Shakir, J. S. Higgins, Colloid Polym. Sci. 1986, 264, 616.

17. K. E. J. Barrett, H. R. Thomas, J. Polym. Sci. Part A: Polym. Chem. 1969, 7, 2621.

18. M. T. Elsesser, A. D. Hollingsworth, Langmuir 2010, 26, 17989.

19. L. Emdadi, C. V. Luciani, S. Y. Lee, I. H. Baick, K. Y. Choi, Polym. Eng. Sci. 2011, 51, 1969.

20. Y. D. Clonis, J. Chromatogr. 1987, 407, 179.

21. K. Cao, J. Yu, B.-G. Li, B. Li, Z. Pan, Chem. Eng. J. 2000, 78, 211.

22. S. Jiang, E. D. Sudol, V. L. Dimonie, M. S. El-Aasser, J. Polym. Sci. Part A: Polym. Chem. 2007, 45, 2105.

23. R. A. Cockburn, T. F. L. McKenna, R. A. Hutchinson, Macromol. React. Eng. 2011, 5, 404. 
24. R. J. Barsotti, L. A. Lewin, C. Scopazzi, U.S. Patent 1998, 5,763,528.

25. W. Wang, R. A. Hutchinson, Macromol. React. Eng. 2008, 2, 199.

26. K. Liang, R. A. Hutchinson, J. Barth, S. Samrock, M. Buback, Macromolecules 2011, 44, 5843.

27. A. C. Simmons, Opt. Commun. 1978, 25, 211.

28. L. Grunberg, A. H. Nissan, Nature 1949, 164, 799.

29. W. J. Blank, Z. A. He, M. Picci, J. Coating Technol. 2002, 74, 33.

30. C. Loubat, S. Soulier, B. Boutevin, Polym. Bull. 2001, 45, 487.

31. K. Cao, B. Li, Z. Pan, Colloids Surf., A 1999, 153, 179.

32. M. A. Parsa, I. Kozhan, M. Wulkow, R. A. Hutchinson, Macromol. Theor. Simul. 2014, 23, 207.

33. T. Tadros, Adv. Colloid Interfac. 2009, 147-148, 281.

34. J. P. A. Heuts, G. E. Roberts, J. D. Biasutti, Aust. J. Chem. 2002, 55, 381.

35. A. N. Nikitin, M. C. Grady, G. A. Kalfas, R. A. Hutchinson, Macromol. React. Eng. 2008, 30, 2022.

36. P. Cacioli, D. G. Hawthorne, R. L. Laslett, E. Rizzardo, D. H. Solomon, J. Macromol. Sci. Chem. 1986, 23, 839. 


\title{
Investigating the Effectiveness of Reactive Dispersants in Non-Aqueous Dispersion Polymerization
}

\author{
Weiwei Yang, Robin A. Hutchinson*
}

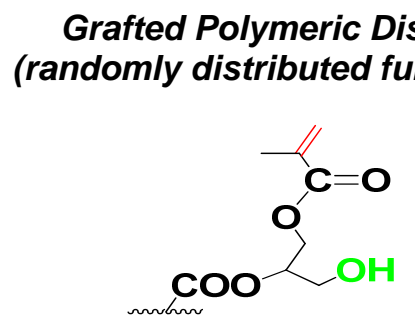

Macromonomer Dispersant

(randomly distributed functionality)

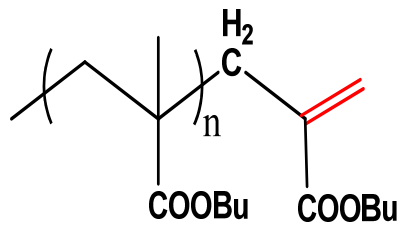

Use of macromer as polymeric dispersant leads to a reduction in particle size of poly(acrylic) nanoparticles produced by semibatch non-aqueous dispersion polymerization. In addition, the fraction of dispersant incorporated into the particle is increased compared to stabilization by randomly functionalized chains. 